\title{
Editorial Note: Large-scale Heterogeneous Multimedia Data Computing and Understanding
}

Multimedia Tools and Applications gratefully acknowledges the editorial work of the scholars listed below on the special issue entitled, "Large-Scale Heterogeneous Multimedia Data Computing and Understanding."

Of 65 papers submitted to this issue, 29 were eventually accepted after a stringent peerreview process.

\section{Zan Gao (Corresponding Guest Editor) \\ Tianjin University of Technology, China \\ zangaonsh4522@gmail.com}

Hanwang Zhang

National University of Singapore, Singapore

hanwangzhang@gmail.com

Charles Wang

MoboPan \&\& The Home Depot, Chicago, USA

mobopan.charles@gmail.com

Yi Yang

University of Technology, Australia

Yi.Yang@uts.edu.au

Publisher's Note Springer Nature remains neutral with regard to jurisdictional claims in published maps and institutional affiliations. 